\title{
Comprehensive Databases: A Cautionary Note
}

\author{
Barry Feig, MD \\ Department of Surgical Oncology, University of Texas MD Anderson Cancer Center, Houston, TX
}

Over the last decade, there has been in an increase in the number and availability of registries of cancer patients. Two of the more commonly accessed sources of cancer patient data are the Surveillance Epidemiology and End Results (SEER) and the National Cancer Data Base (NCDB). These two databases differ fundamentally in how patient data are acquired; the NCDB is hospital based, while SEER is population based. Although this difference in method of data acquisition is extremely different, there are commonalities, both positive and negative, when large data sets such as these are queried to ascertain clinical outcomes on cancer patients. The large number of patients available for analysis allows for a broader perspective on treatment patterns and epidemiology. Additionally, it allows the opportunity to study rare cancers and to perform subset analysis of specific groups within a common cancer (i.e., postmastectomy radiotherapy in patients with locally advanced breast cancer; adjuvant chemotherapy in stage II colon cancer). Most recently, these types of data sets are being used to provide cancer-specific outcomes and minimum performance benchmarks, which are being made available for quality assessment and quality improvement.

Clearly, the quality of the information obtained from these types of investigations depends on accuracy of the data collected. Questions regarding completeness, accuracy, and selection bias are common to both of these databases. It is important to note that the NCDB is a much larger data set than SEER. With increasing size of the data set, there is a greater potential opportunity for inaccuracies. Data are acquired from 1,500 American College of Surgeons Commission on Cancer ( $\mathrm{CoC}$ )-approved hospitals, representing approximately $70 \%$ of all newly diagnosed cancer cases in the United States. ${ }^{1}$ Another notable

(C) Society of Surgical Oncology 2013

Published Online: 19 March 2013

B. Feig, MD

e-mail: bwfeig@mdanderson.org difference between the SEER and NCDB databases has been the issue of user access. The SEER database has had public access - that is, since 1975, it has been available to anyone who had the resources and who was willing to submit a signed usage agreement. ${ }^{2}$ In contrast, access to the NCDB has been limited. Recently, however, the CoC has undertaken a pilot program allowing access to the NCDB data. This access has been limited to investigators at $\mathrm{CoC}$ approved programs who were selected to participate in an alpha test program to evaluate the potential for a more open access program to the NCDB data set. This pilot program is currently in a beta testing phase and is expected to expand to any $\mathrm{CoC}$-approved program sometime in the next year.

As access to large, comprehensive patient databases has increased, so too has the scientific investigation based on this information. There has been a relative explosion of outcomes research based on information obtainable from databases such as SEER and the NCDB. These two frequently used sources of oncologic patient data are maintained by the National Cancer Institute and the Commission on Cancer of the American College of Surgeons, respectively. The comprehensive nature of these databases, along with their large volume of data, almost dictates that they fall under the auspices of a large organization (such as those mentioned), both out of necessity born of the significant resources (financial, personnel, and technical) required, and to permit oversight to maintain standards and accuracy. Additionally, the use of these types of large databases has become so popular that they have spawned a wealth of new societies, degree opportunities, and meeting sections. These have been described by a number of terms including, but not limited to, "health services research," "outcomes research," and "public health research." Along with all of these new meetings, degrees, and societies come new experts in these fields. It appears that as long as there remains access to these type of databases, the number of experts asking questions of these data appears limitless. Unsupervised access to these 
databases has raised a potential concern over inaccurate generalizations that anyone with a pencil and a calculator could be productive if provided access to a large data set. This assumption, though commonly expressed, could not be further from the truth. The complexity of this type of research is often underappreciated, and not infrequently, attempts at large database investigation have been unsuccessful as a result of the investigator's lack of understanding of the tasks involved.

Important lessons have been learned from the NCDB alpha-PUF (Participant User Files) experience. As of one of the alpha-PUF test sites, we have had the opportunity to experience many of these lessons firsthand. The complexity and manpower required to have an effective outcomes research program was readily apparent early on in the alpha-PUF test program. Eighteen months into the program, only a small fraction of the initial six alpha-PUF cancer-specific test sites were able to produce publishable results. This was due, at least in part, to an underestimation on our part (and I would assume a similar view by the other five alpha-PUF sites) of the significant resources that would be required to have a successful program. This includes resources necessary to conduct quantitative analyses, but it also includes a lack of familiarity with the available data elements, making it difficult to ask clinically significant questions that have a reasonable chance of being accurately addressed by the available data. Success in this type of endeavor requires a significant amount of manpower to be productive. We have found it most effective to develop a multidisciplinary clinical outcomes working group. The clinical outcomes team must include participants with clinical expertise in the subject area to ensure that clinically relevant questions are being asked and that these questions have not already been satisfactorily asked and answered in prior publications. A dedicated data manager with experience with the use and structure of large data sets is also a necessity for the accurate and reproducible use of the data set. Incorporating a biostatistician with clinical knowledge as part of the team is another important requisite in order to be successful. A tumor registrar can be extremely helpful in understanding the data fields included in the database.

All of this manpower is expensive and time-consuming. Adequate funding is probably the single biggest obstacle to the success and productivity of a clinical outcomes group. Limited funding resources are available for these endeavors at the federal, state, and local level, despite the recognition of the clinical significance of these types of investigations. Large academic departments are having increasing difficulty finding the funding to support outcomes research as clinical reimbursement dollars continue to shrink. Although prospective randomized trials remain the gold standard for the evaluation of clinical treatment outcomes, the government and private funding agencies need to recognize the value of population-based studies for identifying patterns of biologic behavior, treatment, and outcomes in the context of heterogeneous populations with respect to demographics and socioeconomic variables. With this knowledge, we are able to better understand complex disease processes, which will allow us to design more appropriate and effective prospective trials.

The article by Lerro et al. in the current issue of Annals of Surgical Oncology provides important additional information on the completeness and accuracy of the NCDB as a result of its uniqueness as a hospital-based registry. ${ }^{3}$ The authors, who are members of the $\mathrm{CoC}$ and American Cancer Society staff that maintains the NCDB, display their willingness to openly and fairly examine the accuracy, scope, and completeness of the NCDB. Although previously published works have examined limitations of the NCDB, the current study examines the completeness of NCDB patient case collection by comparing NCDB case counts with case counts from population based registries that report to the United States Cancer Statistics (USCS). ${ }^{4}$ Factors that might affect the variation in completeness of patient case report collection were then analyzed. These factors included geographic variation, ethnic variation, age variation, and disease site. Notably, there were variations in percentage of cases captured that were significant. For example, the overall case coverage by state ranged from a high of $89 \%$ for Delaware to a low of $27 \%$ for Arizona (states with a higher proportion of CoC-approved hospitals will have a higher percentage of cases captured). American Indians and Alaskan Natives were found to have a lower percentage of cases captured compared with whites, blacks, and Asian/Pacific Islanders (32.8\% vs. 64.7-67.4\%). Elderly patients ( $>65$ years) were found to have lower case coverage than persons aged $<65$ (63 vs. $73 \%$ ). Cervical cancer had the highest case coverage (77.9\%), while melanoma had the lowest $(50.6 \%)$. Epidemiologic studies may not be statistically feasible in some of the groups with low case coverage. This type of information will allow investigators using the data from the NCDB to be more critical in statistically validating those factors and groups to be used as variables when designing their outcome studies.

Studies such as the one Lerro et al. are necessary to help improve the accuracy of studies that are generated from large databases. We encourage the staff and administrators of all large data sets to continually assess the accuracy and completeness of their data and not only make this information available, but also ensure that it is acknowledged in ongoing and future studies.

ACKNOWLEDGMENT Disclosure The author declares no conflict of interest. 


\section{REFERENCES}

1. American College of Surgeons. Cancer programs. National Cancer Data Base. http://www.facs.org/cancer/ncdb/.

2. National Cancer Institute. Surveillance Epidemiology and End Results. http://seer.cancer.gov/about/.

3. Lerro CC, Robbins AS, Phillips JL, Stewart AK. Comparison of cases captured in the national cancer data base with those in population-based central cancer registries. Ann Surg Oncol. In press.

4. Bilimoria KY, Bentram DJ, Stewart AK, Winchester DP, Ko CY. Comparison of commission on cancer-approved and -nonapproved hospitals in the United States: implications for studies that use the national cancer data base. J Clin Oncol. 2009;27:4177-81. 